\title{
Distribución del ocelote (Leopardus pardalis) en San Luis Potosí, México
}

\section{Distribution of the ocelot (Leopardus pardalis) in San Luis Potosí, Mexico}

\author{
Jesús Manuel Martínez-Calderas ${ }^{1,2}$, Octavio César Rosas-Rosas ${ }^{1 *}$, Juan Felipe Martínez-Montoya ${ }^{1}$, Luis \\ Antonio Tarango-Arámbula ${ }^{1}$, Fernando Clemente-Sánchez ${ }^{1}$, María Magdalena Crosby-Galván² y Manuel \\ David Sánchez-Hermosillo ${ }^{3}$ \\ ${ }^{1}$ Colegio de Postgraduados, Campus San Luis Potosí. Iturbide 73, 78620 Salinas de Hidalgo, San Luis Potosí, México. \\ ${ }^{2}$ Colegio de Postgraduados. Carretera México-Texcoco Km. 36.5, Montecillo 56230 Texcoco, Estado de México, México. \\ ${ }^{3}$ Secretaría de Desarrollo Agropecuario y Recursos Hidráulicos. Calzada de Guadalupe 1255, Col. Santuario 78380 San Luis Potosí, San Luis Potosí, \\ México. \\ Correspondencia: octaviocrr@colpos.mx
}

\begin{abstract}
Resumen. Para definir la distribución geográfica del ocelote en el estado de San Luis Potosí, México, se obtuvieron nuevos registros de la especie. El estudio se realizó de enero de 2007 a abril de 2009. Se obtuvieron 41 registros de ocelotes por medio de entrevistas y trampeo-fotográfico. Los registros se localizaron en comunidades vegetales de selva baja caducifolia (37\%), matorral submontano (22\%), bosque de encino (15\%), selva mediana (10\%), selva alta perennifolia, bosque mesófilo de montaña, bosque de pino-encino y matorral desértico micrófilo (10\%). La presencia de ocelotes se ubicó en los municipios de Ciudad del Maíz, El Naranjo, Cerritos, Guadalcázar, San Nicolás Tolentino y Ciudad Valles en de elevaciones de 38 a $2400 \mathrm{~m}$ snm. Los resultados de esta investigación sugieren una distribución del ocelote más hacia el oeste del estado respecto a su distribución original. El presente estudio definió nuevas regiones con presencia de ocelotes que pueden ser consideradas en el desarrollo de estrategias de conservación de la especie en el estado de San Luis Potosí.
\end{abstract}

Palabras clave: ocelotes, distribución, San Luis Potosí, entrevistas, comunidad vegetal, elevación, trampeo-fotográfico.

\begin{abstract}
To determine the geographic distribution of ocelot in the state of San Luis Potosí, Mexico, we obtained new records. The study was conducted from January 2007 to April 2009. We recorded 41 ocelot records by interviews and camera-trapping. Ocelots records were located in tropical deciduous forest (37\%), semitropical thornscrub (22\%), oak forest (15\%), tropical forest (10\%), tall tropical deciduous forest, desert scrub, pine-oak forest and clouded forest (10\%). Ocelot records were located in the municipalities of Ciudad del Maíz, El Naranjo, Cerritos, Guadalcazar, San Nicolás Tolentino and Ciudad Valles where the elevation ranged from 38 to $2400 \mathrm{~m}$. The evidence of this research suggests that ocelot range is more extended to the west than its original geographical range. This study defined new regions with presence of ocelots that may be considered to develop conservation strategies for ocelots in San Luis Potosí.
\end{abstract}

Key words: ocelots, distribution, San Luis Potosí, interviews, vegetation community, elevation, camera-trapping.

\section{Introducción}

El ocelote (Leopardus pardalis Linné, 1758) está catalogado por la Norma Oficial Mexicana, NOM-059, 2001 (SEMARNAT, 2002) como una especie en peligro de extinción. Se distribuye desde el sur de Texas en Estados Unidos, México y Centroamérica hasta el norte de Argentina y Uruguay en Sudamérica (Oliveira, 1994; Aranda, 2005). En México, su distribución en las áreas costeras del Pacífico y del golfo de México es continua (Leopold, 1983; Aranda, 2005), desde el oriente de Tamaulipas y el

Recibido: 06 octubre 2009; aceptado: 04 febrero 2011 noroeste de Sonora (Murray et al., 1997), incluyendo los estados de Puebla y Estado de México (Ramírez et al., 2010; O. Monroy-Vilchis, UAEMex, comunicación personal), llegando hasta la península de Yucatán (Aranda, 2005).

En el estado de San Luis Potosí sólo existen 3 registros históricos (Cuadro 1) en colecciones científicas ubicadas en museos de universidades estadounidenses (Dalquest, 1953; López-Wilchis, 2003). Estos registros, aunque no mencionan la ubicación precisa donde se obtuvieron los ejemplares, fueron utilizados por Hall (1981) y Aranda (2005) para definir la distribución inicial de la especie en el estado de San Luis Potosí (Planicie Costera hasta una pequeña porción de la Sierra Madre Oriental). 
Cuadro 1. Registros históricos de ocelotes en San Luis Potosí

\begin{tabular}{|c|c|c|c|c|}
\hline Núm. & Localidad & Ubicación de evidencia & $\begin{array}{c}\text { Elevación de la } \\
\text { localidad ( } \mathrm{m} \mathrm{snm})\end{array}$ & Colector \\
\hline 1 & $\begin{array}{l}\text { Rancho Martínez, } \\
\text { sobre el río Salto, } \\
\text { Ciudad del Maíz }\end{array}$ & $\begin{array}{l}\text { MVZ, University of } \\
\text { California - Berkeley }\end{array}$ & 487.6 & A.S. Leopold \\
\hline 2 & $\begin{array}{l}\text { Hacienda El Limón, } \\
\text { municipio de Ébano }\end{array}$ & $\begin{array}{c}\text { Louisiana State } \\
\text { University. Museum of } \\
\text { Natural Sc. }\end{array}$ & 30 & W.W. Dalquest \\
\hline 3 & $\begin{array}{l}\text { Hacienda El Limón, } \\
\text { municipio de Ébano. }\end{array}$ & $\begin{array}{c}\text { Louisiana State } \\
\text { University. Museum of } \\
\text { Natural Sc. }\end{array}$ & 30 & W.W. Dalquest \\
\hline
\end{tabular}

Los ocelotes suelen encontrarse en una gran variedad de hábitats (Guggisberg, 1975; Oliveira, 1994; Murray et al., 1997), desde bosques tropicales húmedos hasta matorrales secos, siempre y cuando exista vegetación densa (Ludlow y Sunquist, 1987; Oliveira, 1994; Harveson et al., 2004), en elevaciones que van desde el nivel del mar hasta los $3800 \mathrm{~m}$ snm (Vaughan, 1983 en Tewes y Schmidly, 1987; Tello, 1986; Oliveira, 1994), pero preferentemente se les encuentra en elevaciones menores a $1200 \mathrm{~m}$ snm (Nowell y Jackson, 1996). La pérdida de hábitat ocasionada por áreas abiertas a la agricultura, por el desarrollo (i. e. asentamientos humanos e infraestructura de comunicación) y la cacería ilegal, son factores que pueden disminuir las poblaciones de ocelote restringiendo su distribución original. El presente estudio tuvo como objetivo la ubicación de nuevos registros de ocelotes en el estado de San Luis Potosí, México, para definir su distribución y sirva como base para generar planes de conservación a largo plazo para el ocelote.

\section{Materiales y métodos}

La investigación se realizó en el estado de San Luis Potosí, en las zonas conocidas como Zona Media y Huasteca Potosina (Fig.1). El área de estudio se ubica en las subprovincias fisiográficas Llanura Costera, Carso Huasteco y Gran Sierra Plegada entre los paralelos $100^{\circ} 87^{\prime}-98^{\circ} 33^{\prime} \mathrm{O}, 23^{\circ} 30^{\prime}-21^{\circ} 16^{\prime} \mathrm{N}$.

La vegetación del área de estudio se compone de matorral desértico, mezquital, matorral submontano, selva baja caducifolia, selva mediana, selva alta, bosques de encino, pino y sus asociaciones y parches de bosque mesófilo (Rzedowski y Rzedowski, 1957; Rzedowski, 1965; Puig, 1991), así como varios tipos de vegetación inducida, como pastizales y cultivos de caña de azúcar (Rzedowski, 1994). La temperatura media anual es de $26^{\circ} \mathrm{C}$ y la precipitación pluvial anual varía de 700 a $1500 \mathrm{~mm}$, aunque en algunas localidades, entre Xilitla y Tamazunchale, la precipitación es superior a los 2500 $\mathrm{mm}$, y puede alcanzar los $3000 \mathrm{~mm}$ cuando esta área es afectada por ciclones (INEGI, 2002).

El área de estudio ha presentado pérdida de hábitat por diferentes causas; por ejemplo, Villordo et al. (2010) indican que en el periodo 1976-2000 se transformaron 428809 ha de vegetación natural a otros usos de suelo. La población humana en el área de estudio aumentó de 956286 habitantes en 1995 a 1057461 en 2005, aproximadamente 10000 habitantes por año que demandan diversos recursos y servicios (INEGI, 1995, 2005).

El trabajo de campo se realizó de enero de 2007 a abril de 2009 y consistió en recorridos, instalación de trampas-cámara y entrevistas. Los registros fueron clasificados según los criterios propuestos por Tewes y Everett (1986, Cuadro 2), los cuales consideran 3 clases (I, II, III) con base en las evidencias y la credibilidad de cada uno. Los registros clase I son aquellos en los cuales existen evidencias físicas de la presencia de los felinos, tales como pieles, cráneos, huellas, fotografías o avistamientos. En este estudio, los registros clase I se obtuvieron por medio de trampas-cámara y recorridos de campo, observando huellas y rastros, de acuerdo con Aranda (2000). Los registros clase II y III se ubicaron por medio de entrevistas. La clase II considera información detallada y verosímil sobre el avistamiento de ocelotes. Los registros clase III corresponden a situaciones en las que no es posible discernir si se trata de un ocelote u otra especie, debido a que los datos aportados por el entrevistado son insuficientes para la identificación correcta. Por ello, sólo se tomaron en cuenta para el análisis los registros clase I y II.

El uso de trampas-cámara es un método no intrusivo que ayuda a confirmar la presencia de especies elusivas, y se ha empleado para determinar la presencia y estimar la distribución y abundancia de varias especies (Karanth, 1995; Karanth y Nichols, 1998; Maffei et al., 2004; Silver et al., 2004; Trolle y Kery, 2003; Trolle y Kery, 2005; 
Cuadro 2. Clases de registro de ocelotes para evaluar su credibilidad. Tomado de Tewes y Everett (1986) Las clases I y II son las que se consideran en el presente estudio

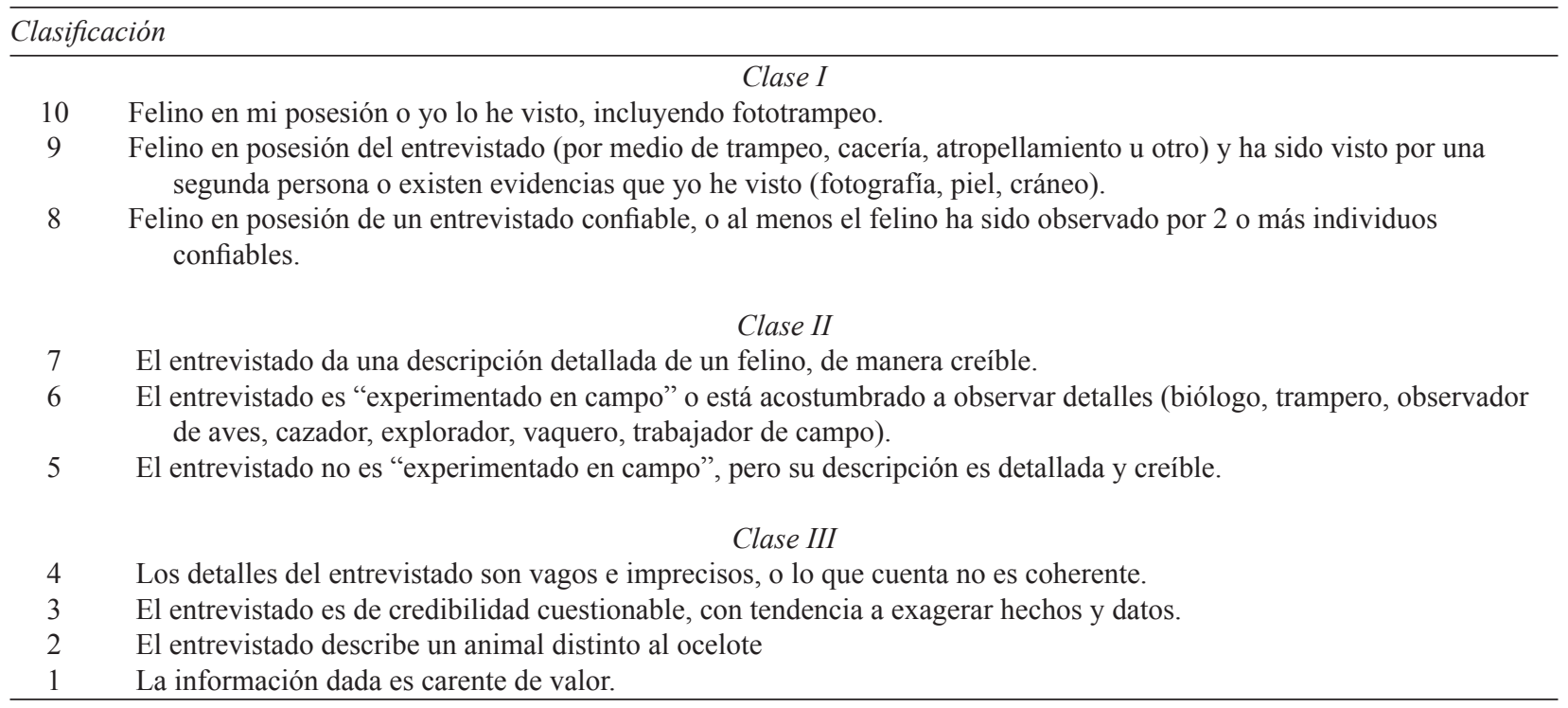

Sanderson y Trolle, 2005). En este estudio se utilizaron 10 trampas-cámara Camtrakker@) (Watkinsville, Georgia, USA) instaladas en sitios donde se encontraron posibles evidencias o registros de la presencia de estos felinos. Las trampas fotográficas se colocaron a una altura de 50 $\mathrm{cm}$ de la superficie del suelo, y permanecieron activas constantemente por periodos de 20 días, durante 2 meses en 2007 y en 2008, respectivamente, en sitios donde se había informado de la presencia de la especie. Los sensores se configuraron para tomar fotografías con un retraso mínimo de 5 minutos entre cada foto.

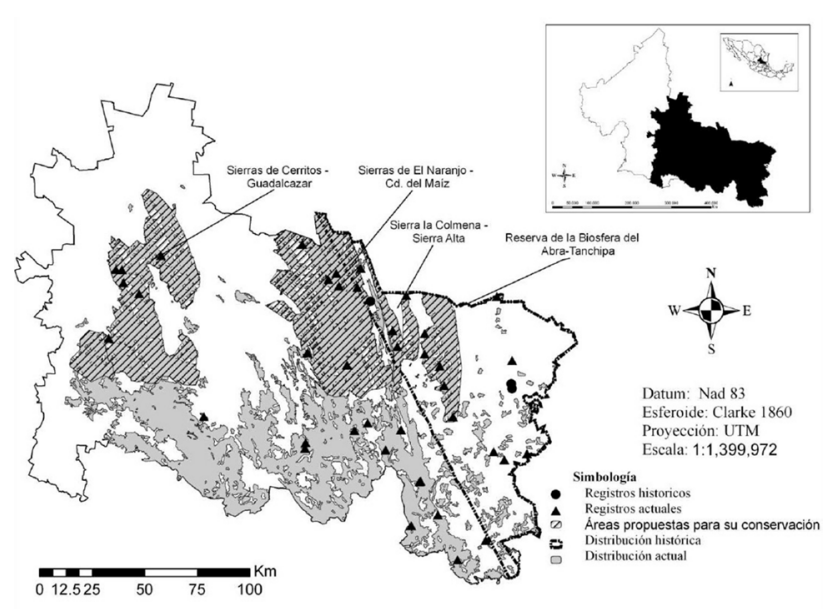

Figura 1. Área de estudio y distribución propuesta para el ocelote en San Luis Potosí.
También se utilizaron trampas de pelo como método alternativo para corroborar la identificación de la especie (MacDaniel et al., 2000; Weaber et al., 2005). Las trampas de pelo se colocaron a $3 \mathrm{~m}$ de las cámaras sobre troncos 0 postes de cercos, a una altura de $40 \mathrm{~cm}$ de la superficie del suelo. Asimismo, se utilizaron estaciones atrayentes para maximizar la atracción de ocelote colocándose a $150 \mathrm{~cm}$ de distancia de las cámaras (Linhart y Knowlton, 1975). Estas estaciones fueron de $1 \mathrm{~m}$ de diámetro, elaboradas con tierra cernida y como atrayentes se utilizaron alternativamente orines de ocelote en cautiverio, Catnip en aerosol $(\operatorname{Cosmos} \odot)$ y aceite de sardina; cada uno se mezcló con tierra en el centro de cada estación.

Como primera fase, se concertaron entrevistas con funcionarios municipales para ubicar a personas que tuvieran experiencia de campo y capacidad para identificar correctamente la morfología de la especie. Con esta información, se entrevistó a peleteros, cazadores (no ejidatarios), veterinarios, ganaderos (no ejidatarios) y ejidatarios radicados en la zona de estudio. Las entrevistas se desarrollaron como conversaciones informales, sin tomar apuntes en el momento de su realización, para minimizar sesgos por desconfianza de los entrevistados, especialmente en comunidades indígenas donde fue notoria su renuencia a aportar información. En las entrevistas se requirieron datos sobre lugar de avistamiento, sitios de paso común de ocelotes (con base en rastros u observaciones recurrentes) y si se tenía conocimiento de personas que estuvieran en posesión de pieles de animales. Una vez obtenida la información y con el apoyo de guías, en su mayoría los mismos informantes, se proce- 
dió a visitar los sitios específicos donde los entrevistados mencionaron presencia de ocelotes, para registrar la ubicación geográfica y el tipo de vegetación. Los registros se georreferenciaron con un geoposicionador (GPS, Garmin ( $)$ modelo GPS V).

Para definir el mapa de distribución (Fig. 2) se utilizaron las coordenadas geográficas, el tipo de registro, y el tipo y cobertura de la vegetación de cada uno de los registros. Con la ayuda de ArcViewC (9.2, se ubicaron los registros en el mapa de vegetación del área de estudio. Para llevar a cabo esta ubicación, se consideró una distancia de $4 \mathrm{~km}$ de buffer de cada registro (distancia mínima de actividad del ocelote, Crawshaw, 1995; Dillon y Kelly, 2007), las características del hábitat (tipos de vegetación y cobertura de la misma), y visualmente se analizaron imágenes Landsat del año 2000 en falso color (INEGI, 2008, http:// mapserver.inegi.org.mx) y de la Secretaría de Desarrollo Agropecuario y Recursos Hidráulicos del Estado de San Luis Potosí (SEDARH, 2007). Cabe señalar que en las entrevistas no se siguió un esquema de distribución por tipo de vegetación, éste lo determinó el entrevistador una vez definidos los sitios de registro.

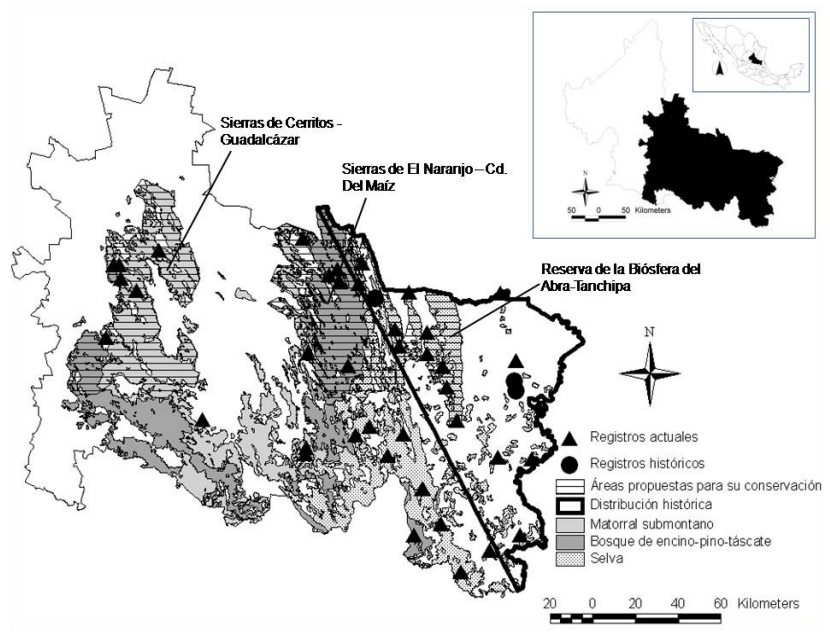

Figura 2. Tipos de vegetación dentro del área de distribución propuesta del ocelote (Leopardus pardalis) en San Luis Potosí, México.

\section{Resultados}

Se realizaron 37 salidas de campo (4 a 7 días/salida) y 270 entrevistas en comunidades y ejidos (120 en la Zona Media y 150 en la Huasteca Potosina). De las 270 entrevistas, 28 fueron con funcionarios municipales, 6 con peleteros, 12 con cazadores, 3 con veterinarios y 221 con ganaderos y ejidatarios de ambas zonas. Del total de entrevistas se obtuvieron 41 registros de ocelotes (14 clase I y 27 clase II; Cuadro 3). De éstos, 7 correspondie- ron a datos históricos entre 20 y 35 años de antigüedad, evidenciados con fotografías, pieles y avistamientos. Los registros clase I consistieron en 6 fotografías, 6 pieles, 1 ejemplar vivo (decomisado por la Procuraduría Federal de Protección al Ambiente) y 1 ejemplar identificado por huellas. El resto de las entrevistas se clasificaron como clase III.

El esfuerzo de trampeo-fotográfico comprendió 4 meses en el periodo de 2007-2008 ( 2 meses en 2007 y 2 meses en 2008 incluyendo 1 mes de época seca y 1 mes de época húmeda en cada año), dando un total de 800 noches/trampa y 4 fotografías correspondientes a diferentes individuos de la especie.

Las trampas para pelo no fueron útiles para obtener registros de ocelotes, y las estaciones atrayentes, si bien, fueron efectivas para las especies como margay (Leopardus wiedii), armadillos (Dasypus novemcinctus) y zorras (Urocyon cinereoargenteus), no lo fueron para el ocelote.

En su totalidad, los registros se localizaron en los siguientes tipos de vegetación (Cuadro 3): selva baja caducifolia $(37 \%)$, matorral submontano $(22 \%)$, bosque de encino (15\%), selva mediana (10\%), selva alta perennifolia, bosque mesófilo de montaña, bosque de pino-encino y matorral desértico micrófilo (10\%).

De estos registros, 3 corresponden a individuos cazados hace 30 años; son los que registran Hall (1981) y Aranda (2005) cuando se refieren a la distribución de la especie en San Luis Potosí. No fue posible confirmar el tipo de vegetación debido al cambio de uso de suelo y por la falta de relictos de vegetación en la zona.

La mayoría de los registros (76\%) se ubicaron por debajo de los $1200 \mathrm{~m}$ snm, los otros corresponden a elevaciones dentro del intervalo de $1241-2400 \mathrm{~m}$ snm. El registro con menor altitud ( $38 \mathrm{~m} \mathrm{snm}$ ) se localizó en selva baja caducifolia en el municipio de San Vicente Tancuayalab (Planicie Costera) y el más elevado (2 $400 \mathrm{~m} \mathrm{snm})$ en La Trinidad, Xilitla, en vegetación de pino-encino. (Cuadro 3).

Con base en los registros nuevos e históricos se determinó que la distribución del ocelote en San Luis Potosí incluye los municipios de Ébano, San Vicente Tancuayalab, Tamuín, Ciudad Valles, Aquismón, Tampacán, Huehuetlán, Tamazunchale, Xilitla, Tamasopo, Cd. del Maíz, Alaquines, Rayón, Rioverde, Lagunillas, Cerritos, Guadalcázar, San Nicolás Tolentino y el Naranjo (Fig. 1).

\section{Discusión}

Para determinar la distribución del ocelote en el estado de San Luis Potosí se tuvieron entrevistas con habitantes de las comunidades y ejidos, se instalaron trampas fotográficas apoyadas con trampas de pelo, así 
Cuadro 3. Registros recientes de ocelotes en San Luis Potosí donde X y Y corresponden a las coordenadas UTM, NAD 83

\begin{tabular}{|c|c|c|c|c|c|c|c|}
\hline Localidad & Tipo de registro & Clase & $Y$ & $X$ & $m \mathrm{snm}$ & Vegetación & $A \tilde{n} o$ \\
\hline La Trinidad, Xilitla. & Fotografía & I & 2367767 & 489905 & 2400 & BPQ & 2006 \\
\hline Papagayos, Ciudad del Maíz. & Fotografía & I & 2481805 & 455479 & 1800 & BMM & 2006 \\
\hline Tanchanchín, Valles. & Fotografía & $\mathrm{I}$ & 2413513 & 484988 & 138 & $\mathrm{SM}$ & 2006 \\
\hline El Otate, Tamazunchale. & Entrevista, caza & II & 2351532 & 511994 & 985 & SAP & 2007 \\
\hline San Vicente Tancualayab. & Piel & I & 2402047 & 545018 & 38 & ND & Circa 1987 \\
\hline Joya de Luna, Cerritos. & Entrevista, caza & II & 2489612 & 352168 & 1472 & MS & Circa 1972 \\
\hline Rincón de Banda, Cerritos. & Entrevista, avistamiento & II & 2478195 & 360346 & 1300 & MS & 2004 \\
\hline Joya de Luna, Cerritos. & Entrevista, avistamiento & II & 2489631 & 349281 & 1510 & MS & 2008 \\
\hline Joya de Luna, Cerritos. & Entrevista, caza & II & 2483385 & 352888 & 1241 & MS & 2008 \\
\hline El Encanto, Tamuín. & Caza & I & 2446404 & 537892 & 49 & SBC & 2007 \\
\hline Ejido Paso. Prieto, Rayón. & Entrevista, atropellado & II & 2407273 & 439655 & 851 & MS & Circa 1978 \\
\hline Ejido López Mateos, Valles & Fotografía de caza & $\mathrm{I}$ & 2419512 & 509846 & 150 & SBC & Circa 2000 \\
\hline $\begin{array}{l}\text { San Francisco Cuayalab, San Vicente } \\
\text { Tancualayab. }\end{array}$ & Piel & I & 2403015 & 529101 & 76 & SBC & Circa 2000 \\
\hline $\begin{array}{l}\text { San Francisco Cuayalab, San Vicente } \\
\text { Tancualayab. }\end{array}$ & Piel & I & 2367807 & 539821 & 76 & SBC & 2008 \\
\hline Las Lajas, Aquismón. & Entrevista, avistamiento & II & 2388729 & 493840 & 468 & SM & 2008 \\
\hline El Naranjo. & Huella & I & 2481197 & 464140 & 260 & BQ & 2006 \\
\hline San José del Viejo, Tamasopo. & Decomiso & I & 2403837 & 477689 & 638 & SM & 2008 \\
\hline El Naranjo. & Piel & I & 2490330 & 465829 & 270 & ND & ND \\
\hline El Naranjo. & Piel & I & 2490330 & 465829 & 270 & ND & ND \\
\hline El Nacimiento I, Huehuetlán. & Entrevista, avistamiento & II & 2372930 & 502545 & 120 & SBC & 2008 \\
\hline Ejido La Ceiba, Tampacán. & Entrevista, avistamiento & II & 2360834 & 525343 & 152 & SBC & 2008 \\
\hline San Nicolás de los Montes, Tamasopo. & Fotografía & $\mathrm{I}$ & 2444342 & 459364 & 800 & BQ & 2008 \\
\hline Pozo de Acuña, Guadalcázar. & Entrevista, avistamiento & II & 2496284 & 370786 & 1353 & MS & 2009 \\
\hline Las Lajas, Aquismón. & Entrevista, avistamiento & II & 2388917 & 494376 & 448 & $\mathrm{SM}$ & 2009 \\
\hline Ejido León Guzmán, Valles. & Entrevista, avistamiento & II & 2434498 & 505509 & 211 & SBC & 2009 \\
\hline Los Sabinitos 2, Valles. & Entrevista, avistamiento & II & 2443634 & 503587 & 230 & SBC & 2009 \\
\hline Ejido Laguna del Mante, Valles. & Entrevista, avistamiento & II & 2459206 & 496337 & 202 & SBC & 2009 \\
\hline Ejido Las Pitas, Valles. & Entrevista, avistamiento & II & 2477377 & 487586 & 267 & SBC & 2009 \\
\hline El Jabalí, Rioverde. & Fotografía de prensa & $\mathrm{I}$ & 2419802 & 391151 & 1170 & MS & 2009 \\
\hline Santa Marta, Ébano & Entrevista, avistamiento & II & 2476859 & 530635 & 30 & SBC & 2009 \\
\hline El Cañón, Lagunillas. & Entrevista, avistamiento & II & 2404585 & 439350 & 764 & MS & 2009 \\
\hline Cabezas, Tamasopo. & Entrevista, avistamiento & II & 2416684 & 469137 & 450 & SBC & 2009 \\
\hline Santa María Tampalatín, Tamasopo. & Entrevista, avistamiento & II & 2413117 & 462834 & 628 & BQ & 2009 \\
\hline Cerro alto, Valles. & Entrevista, avistamiento & II & 2449497 & 496296 & 146 & SBC & 2009 \\
\hline La Estribera-La Perla, Valles. & Entrevista, avistamiento & II & 2453038 & 483238 & 480 & SBC & 2008 \\
\hline Puerta del Espíritu Santo, El Naranjo. & Entrevista, avistamiento & II & 2460683 & 481006.14 & 520 & SBC & 2009 \\
\hline El Platanito, El Naranjo. & Entrevista, avistamiento & II & 2487873 & 454186 & 1058 & BQ & Circa 1994 \\
\hline Abra de Caballeros, El Naranjo. & Entrevista, atropellado & II & 2484924 & 450435 & 1119 & BQ & 2009 \\
\hline San Juan del Meco, Cd. del Maíz. & Entrevista, avistamiento & II & 2501638 & 437983 & 1300 & BQ & 2008 \\
\hline Tortuga, Alaquines. & Entrevista, avistamiento & II & 2450050 & 440442 & 1480 & MDM & Circa 2008 \\
\hline Ojo de agua, San Nicolás Tolentino. & Entrevista, caza & II & 2456942 & 345869 & 1640 & MS & 2007 \\
\hline
\end{tabular}

BPQ, bosque de pino-encino; BQ, bosque de encino; BMM, bosque mesófilo de montaña; MDM, matorral desértico micrófilo; MS, matorral submontano; SAP, selva alta perennifolia; SBC, selva baja caducifolia; SM, selva mediana; ND, no determinado.

como estaciones atrayentes.

Es posible que por una errónea identificación de las especies, la información sobre la fauna silvestre que proporcionan los entrevistados sea incorrecta, pero esta situación no ocurrió en el presente estudio por 2 razones: 1), los registros que se utilizaron para definir la distribu- ción de los ocelotes, fueron clasificados en clase I y clase II. Estas clases obligan al recopilador de información a verificarlos, mediante fotografías, pieles o ejemplares disecados, entre otras evidencias y 2), la posibilidad de confundir los ocelotes con jaguares juveniles es baja, debido a que la presencia de éstos siempre está aso- 
ciada a la de sus madres; además, las huellas de jaguares juveniles generalmente son de mayor tamaño que las de ocelotes adultos (Aranda, 2000). La posible confusión con el tigrillo es de igual manera baja, por las características morfológicas diferentes, en especial el tamaño de la cola, ojos y patrones de manchas de cada especie, y sobre todo por el tamaño más grande de la huella del ocelote (Aranda, 2000).

Las trampas para pelo no fueron útiles para apoyar el trampeo fotográfico y tampoco las estaciones atrayentes que sí fueron efectivas con otras especies, como L. wiedii, D. novemcinctus y $U$. cinereoargenteus. Es posible que el tipo de atrayente y las condiciones ambientales, en ese orden, hayan afectado la capacidad de percepción de esta especie.

La mayoría de los registros de ocelotes se ubicaron en selva baja caducifolia, matorral submontano y bosque de encino (Fig. 2). La selva baja caducifolia y el matorral submontano comúnmente se registran como hábitat del ocelote (Guggisberg, 1975; Leopold, 1983; Vaughan, 1983; Tewes y Schmidly, 1987), en tanto que el bosque de encino sólo lo mencionan Navarro (1985) y Brown (1989) como hábitat en Texas. En México, la presencia del ocelote no se había registrado en el bosque de pinoencino ni en el matorral desértico micrófilo. Este estudio muestra las primeras evidencias del ocelote en estos tipos de vegetación (Cuadro 3).

Los registros de ocelotes se ubicaron por debajo de los $1200 \mathrm{~m}$ snm, concordando con lo registrado por Vaughan (1983), Tewes y Schmidly (1987) y Nowell y Jackson (1996). Este estudio indicó que en San Luis Potosí, el ocelote se distribuye en la selva baja de la Planicie Costera al oriente del estado, en la Sierra Madre Oriental y la Huasteca Potosina, extendiéndose hacia el oeste hasta los macizos montañosos de la Zona Media. La distribución del ocelote en el estado de San Luis Potosí obtenida en este estudio complementa la definida por Hall (1981) y Aranda (2005) y permite incluir con fines de manejo y conservación de la especie, zonas no consideradas en la distribución original.

Durante este estudio no se encontraron evidencias de que el ocelote se distribuya más allá de la Zona Media hacia el Altiplano Potosino y el este de la Sierra Madre Oriental en su Planicie Costera (Fig. 1). Los resultados de este trabajo definen las fronteras de su distribución: al norte, Ciudad del Maíz; al sur, los municipios de Xilitla y Rayón; al este, la Reserva de la Biosfera Sierra Abra-Tanchipa en el municipio de Valles, y al oeste los municipios de Cerritos y Guadalcázar.

Es poco probable que el ocelote habite las regiones fuera de la Zona Media y Sierra Madre Oriental, las cuales presentan climas más áridos con temperaturas más extremas y vegetación desértica menos densa, y más frecuencia de asentamientos humanos. La combinación de estos factores hace a estas regiones inadecuadas para la presencia de ocelotes.

De acuerdo con este estudio, la Reserva de la Biosfera del Abra-Tanchipa, la Sierra de La Colmena en la región de El Naranjo y las sierras de los municipios de Cerritos y Guadalcázar se proponen como regiones importantes de distribución, manejo y conservación del ocelote (Fig. 1). Estas áreas mantienen una vegetación densa y su estructura es apropiada para la protección de la especie pudiendo albergar una población viable a largo plazo. La distribución del ocelote en San Luis Potosí (este estudio) se puede complementar con estudios similares en los estados de Tamaulipas, Veracruz, Hidalgo y Querétaro con la finalidad de establecer estrategias regionales de manejo y conservación de la especie.

\section{Agradecimientos}

Al Consejo Nacional de Ciencia y Tecnología, por la beca de maestría de Jesús Manuel Martínez-Calderas; a la Procuraduría Federal de Protección al Ambiente; al Dr. Jorge Palacio Núñez, por las sugerencias al manuscrito; a todas las personas que de una $u$ otra manera contribuyeron y ofrecieron información durante el desarrollo del presente trabajo.

\section{Literatura citada}

Aranda, M. 2000. Huellas y otros rastros de mamíferos grandes y medianos de México. Instituto de Ecología, Xalapa, Veracruz. 212 p.

Aranda, M. 2005. Ocelote. In Los mamíferos silvestres de México, G. Ceballos y G. Oliva (eds.). FCE/ CONABIO, México, D. F. p. 359-361.

Brown, D. E. 1989. The ocelot. In Audubon wildlife report, W. J. Chandler (ed.). Harcourt Brace Jovanovich, San Diego, California. p. 421-433.

Crawshaw, P. G. 1995. Comparative ecology of ocelot (Felis pardalis) and jaguar (Panthera onca) in a protected subtropical forest in Brazil and Argentina. PhD. Thesis. University of Florida, Gainsville. 190 p.

Dalquest, W. W. 1953. Mammals of the Mexican state of San Luis Potosí. Lousiana State University Press, Baton Rouge. 229 p.

Dillon, A. y M. T. Kelly. 2007. Ocelot radio telemetry: ocelot trap success, activity patterns, home range and density. Oryx 41:469-477.

Guggisberg, C. A. W. 1975. Wild cats of the world. Taplinger, New York. 328 p.

Hall, E. R. 1981. The mammals of North America, segunda 
edición. Wiley, New York. 1181 p.

Harverson P. M, M. E. Tewes, G. L. Anderson y L. L. Laack. 2004. Habitat use by ocelots in south Texas, implications for restoration. Wildlife Society Bulletin 32:948-954.

INEGI (Instituto Nacional de Geografia e Informática).1995. I Conteo de población y vivienda. INEGI, Aguascalientes, Aguascalientes. http://www.inegi.org.mx/inegi/default. aspx?s=est\&c=10202; última consulta: 05.VI.2010.

INEGI (Instituto Nacional de Geográfia e Informática). 2002. Síntesis de información geográfica del estado de San Luis Potosí. Aguascalientes, Aguascalientes.

INEGI (Instituto Nacional de Geografía e Informática). 2005. I Conteo de población y vivienda. INEGI, Aguascalientes, Aguascalientes. http://www.inegi.org.mx/inegi/default. aspx?s=est\&c=10202; última consulta: 05.VI.2010.

INEGI (Instituto Nacional de Geografía e Informática). 2008. Continuo de elevación nacional. http://mapserver.inegi.org. mx; última consulta: 05.VI.2010.

Karanth, K. U. 1995. Estimating tiger Panthera tigris populations from camera trap data using capture-recapture models. Biological Conservation 71:333-338.

Karanth, K. U. y J. D. Nichols. 1998. Estimation of tiger densities in India using photographic captures and recaptures. Ecology 79:2852-2862.

Leopold, A. S. 1983. Fauna silvestre de México. Pax-México. $655 \mathrm{p}$.

Linhart, S. B. y F. F. Knowlton. 1975. Determining the relative abundance of coyotes by scent station lines. Wildlife Society Bulletin 3:119-124.

López-Wilchis, R. 2003. Base de datos de los mamíferos de México depositados en colecciones de Estados Unidos y Canadá. Universidad Autónoma Metropolitana-Iztapalapa. http://investigacion.izt.uam.mx/mamiferos/última consulta: 22.VIII.2008

Ludlow, M. E. y M. E. Sunquist. 1987. Ecology and behavior of ocelots in Venezuela. National Geographic Research 3:447461.

Maffei, L., E. Cuellar y A. Noss. 2004. One thousand jaguars (Panthera onca) in Bolivia's Chaco? Camera trapping in the Kaa-Iya National Park. Journal of Zoology 262:295304.

Martínez-Calderas, J. M. 2009. Nuevos registros y distribución del ocelote (Leopardus pardalis) en el Noreste de México. Tesis Maestría en Ciencias, Colegio de Postgraduados. 96 p.

McDaniel, Q. W., K. S. Mckelvey, J. R. Squires y L. F. Ruggerio. 2000. Efficacy of lures and hair snares to detect lynx. Wildlife Society Bulletin 28:119-123.

Murray, J. L. y G. L. Gardner. 1997. Leopardus pardalis. Mammalian Species 548:1-10

Navarro, L. D. 1985. Status and distribution of the ocelot (Felis pardalis) in south Texas. M.Sc. Thesis, Texas A\&I
University, Kingsville, Texas. 92 p.

Nowell, K. y P. Jackson.1996. Wild cats: status survey and conservation action plan. UICN, Gland. 382 p.

Oliveira, T. G. De. 1994. Neotropical cats: ecology and conservation. Edufma, Säo Luís Maranhão. 220 p.

Puig, H. 1991. Vegetación de la Huasteca (México), estudio fitogeográfico y ecológico. Instituto de Ecología / CEMCA/ Institut Francaise de Recherche Scientifique Pour le Developpement en Cooperation, Xalapa, Veracruz. 625 p.

Ramírez, B. O., S. B. Schinkel y C. S. Hernández. 2010. Nuevo registro del ocelote (Leopardus pardalis) para el estado de Puebla. Therya 1:91-94

Rzedowski, J. y G. C. de Rzedowski. 1957. Notas sobre la vegetación de San Luis Potosí. V. La vegetación a lo largo de la carretera San Luis Potosí-Río Verde. Acta Científica Potosina 1:7-68

Rzedowski, J. 1965. Vegetación del estado de San Luis Potosí. Acta Científica Potosina 5:5-291.

Rzedowski, J. 1994. Vegetación de México. Limusa, México, D.F. 432 p.

Sanderson, J. G. y M. Trolle. 2005. Monitoring Elusive Mammals Unattended. Cameras reveal secrets of some of the world's wildest places. American Scientist 93:148-155

SEDARH (Secretaría de Desarrollo Agropecuario y Recursos Hidráulicos). 2007. Mapa base del estado de San Luis Potosí. San Luis Potosí, San Luis Potosí.

SEMARNAT. (Secretaría del Medio Ambiente y Recursos Naturales). 2002 Norma Oficial Mexicana, 2002. NOM059-ECOL-2001, Protección ambiental-Especies nativas de México de flora y fauna silvestres-Categorías de riesgo y especificaciones para su inclusión, exclusión o cambioLista de especies en riesgo. Diario Oficial de la Federación, 6 de marzo.

Silver, C. S., L. E. T. Ostro, L. K. Marsh, L. Maffeil, A. J. Noss, M. Kelly, R. B. Wallace, H. Gómez y G. Ayala. 2004.

Tello, J. L. 1986. The situation of the wild cats (Felidae) in Bolivia. CITES Secretariat, Lausanne. 76 p.

Tewes, M. E. y D. Everett. 1986. Status and distribution of the endangered ocelot and jaguarondi in Texas. In Cats of the world: biology, conservation, and management, S. D. Miller y D. D. Everett (eds.). National Wildlife Federation, Washington, D.C. p. 147-158.

Tewes, M. E. y D. J. Schmidly, 1987. The neotropical felids: jaguar, ocelot, margay, and jaguarondi. In Wild furbearer management and conservation in North America, M. Novak, J. A. Baker, M. E. Obbard y B. Malloch (eds.). Ontario Ministry of Natural Resources, Toronto. p. 697-711.

Trolle, M. y M. Kery. 2003. Estimation of ocelot density in the Pantanal using capture-recapture analysis of cameratrapping data. Journal of Mammology 84:607-614.

Trolle, M. y M. Kery. 2005. Camera-trap study of ocelot and other secretive mammals in the northern Pantanal. 
Mammalia 69:405-412.

Vaughan, C. 1983. A report on dense forest habitat for endangered wildlife species in Costa Rica. National University, Heredia, Costa Rica. 99 p.

Villordo-Galván, J. A., O. C. Rosas-Rosas, J. F. MartínezMontoya, F. Clemente-Sánchez, L. A. Tarango-Arámbula,
M. D. Sánchez-Hermosillo y L. C. Bender. 2010. The jaguar (Panthera onca) in San Luis Potosí, México. The Southwestern Naturalist 55:394-402.

Weaber, J. L., P. Wood, D. Paetkau y L. L. Laack. 2005. Use of scented hair snares to detect ocelots. Wildlife Society Bulletin 33:1384-1319. 